\title{
Analysis of High-Altitude Ionization Gauge Measurements Using the Direct Simulation Monte Carlo Method
}

\author{
Quanhua Sun ${ }^{*}$, Chunpei Cai ${ }^{\dagger}$, and Iain D. Boyd* \\ University of Michigan, Ann Arbor, MI 48109 \\ James H. Clemmons ${ }^{\S}$ and James H Hecht ${ }^{* *}$ \\ The Aerospace Corporation, EI Segundo, CA 90245
}

\begin{abstract}
The rarefied, three-dimensional flows experienced during the Turbulent Oxygen Mixing Experiment (TOMEX) at altitudes between $85 \mathrm{~km}$ and $143 \mathrm{~km}$ are simulated using the direct simulation Monte Carlo (DSMC) method. The present study focuses on ionization gauge measurements obtained by TOMEX. The payload is modeled in detail and several complex meshes are generated for the simulations. The simulations show that a bow shock wave is generated in front of the payload at low altitude that becomes diffusive at higher altitudes. When the altitude increases, the pressure in the channels of the ionization gauge and the pressure variation around the payload are both decreased. The DSMC results agree very well with data predicted by compressible flow theory and free molecular flow theory when applicable. Comparison between the DSMC results and the TOMEX flight data shows generally good agreement.
\end{abstract}

\section{Introduction}

$\mathrm{A}$ number of chemical and dynamical processes that have minor significance throughout much of the atmosphere play a major role in determining the structure and energetics at altitudes between $80 \mathrm{~km}$ and $110 \mathrm{~km}$ that is often referred to as the MALT (Mesosphere and Lower Thermosphere) region. Such processes include the production and diffusion of atomic oxygen, interactions of waves and tides, and breakdown of atmospheric gravity waves (AGWs). While the relationship between eddy diffusion, turbulence, instability structures, dynamical activity, and constituent transport, has been often discussed and speculated upon, an important question is how the species present in the MALT region are actually affected when potentially unstable conditions occur.

The Turbulent Oxygen Mixing Experiment (TOMEX) ${ }^{1}$ was designed to measure the actual atmospheric response to the existence of unstable layers as determined by wind and temperature measurements from $80 \mathrm{~km}$ to $105 \mathrm{~km}$. The TOMEX combined Na lidar measurements, from Starfire Optical Range in Albuquerque, New Mexico, with a launch of a mother-daughter payload from White Sands Missile Range, located a little over 100km from Starfire. The payload included a trimethyl aluminum (TMA) release (on the mother payload) to measure winds and diffusion, a five-channel ionization gauge (IG) (on the daughter payload) to measure neutral density fluctuations at high vertical resolution, and a three-channel photometer experiment (on the daughter payload) to measure atomic oxygen related airglow. The payload was launched at 0957 UT on 26 October 2000 and successfully obtained data from all the experiments.

The neutral density measurement employed a five-channel ionization gauge. Each channel consisted of a miniature sensor and an accommodation chamber. The ionization gauge was sealed before launch and opened by breaking the seal once the payload attained altitude. Electronics inside the payload provided the circuitry to control and measure the emitted electron current as well as to measure the collected ion current. High-resolution, high-speed analog-to-digital converters were used in order to provide rapid $(1 \mathrm{kHz})$ measurements spanning a large dynamic range in pressure. In order to obtain the pressure (density) distribution around the payload, one channel was placed forward-looking in the front of the payload, whereas the other four were equally spaced around the payload.

\footnotetext{
* Post-Doctoral Research Fellow, Department of Aerospace Engineering, Member AIAA.

${ }^{\dagger}$ Graduate Student Research Assistant, Department of Aerospace Engineering.

* Professor, Department of Aerospace Engineering, Associate Fellow AIAA.

${ }_{*}^{\S}$ Research Scientist, Space Science Applications Laboratory, M2-260, P.O.Box 92957, Los Angeles, CA 90009

${ }^{* *}$ Senior Scientist, Space Science Applications Laboratory, M2-259, P.O.Box 92957, Los Angeles, CA 90009
} 
The density measurement using the five-channel ionization gauge can infer the flow density, temperature and velocity, in principle. The complexity of the flow, however, can make such interpretation difficult. For instance, there were momentum and energy transfer on the payload surface. A bow shock wave was formed in front of the payload. The mean free path of the atmosphere in the MALT region was comparable to the payload size, which indicates that the flow was in the transitional or even free molecular regime. There is no simple theory that can account for the complicated flow experienced during the TOMEX. Numerical simulations are therefore necessary to interpret the measurement data.

The objective of this paper is to help understand the flow experienced during the TOMEX using numerical simulations. Additional findings from the TOMEX can be found in several other papers. ${ }^{2-5}$ In this paper, the payload geometry is modeled (section II) using some necessary simplifications. Flows in and around the payload are simulated (section III) using the direct simulation Monte Carlo (DSMC) method, which is an efficient kinetic approach widely used in simulating rarefied gas flows. Simulated results are then analyzed and compared with the measurement data (section IV) followed by some concluding remarks (section V).

\section{Payload Modeling}

During the TOMEX experiment, the daughter payload was ejected above $65 \mathrm{~km}$ at a separation of a few meters per second. It traveled at about $1 \mathrm{~km} / \mathrm{s}$ through the $80 \mathrm{~km}$ to $110 \mathrm{~km}$ region with a spin rate of almost exactly $1 \mathrm{~Hz}$. The payload has a cylindrical surface with several instruments installed inside and outside as shown in Fig. 1. On the top of the payload are three photometer telescopes and a channel of the ionization gauge. There are four equally placed openings on the side surface of the payload where each opening faces one channel of the ionization gauge. Three deck plates are used to mount the internal instruments. Flow is negligible through the upper and lower deck plates, whereas the cutouts in the middle deck plate take about half of the deck plate area. The regions between the upper and lower deck plates are the only internal part of the payload having significant flow from the atmosphere. The ionization gauge is an instrument that provides a measurement of the atmospheric neutral density and its variations. The technique is based on a simple principle: a volume of gas to be measured is partially ionized by a stream of electrons, then the resulting ions are collected by a biased collector. The gas density can be determined because it is proportional to the ratio of the emitted electron current to the collected ion current. Figure 2 shows a schematic view of a channel of the ionization gauge where the vacuum door is opened during the measurement.

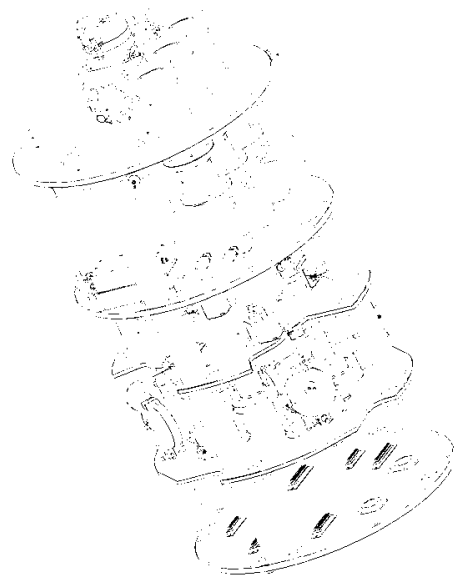

Figure 1. The Aerospace Corporation 3-Channel Photometer and Ionization Gauge Daughter Payload.

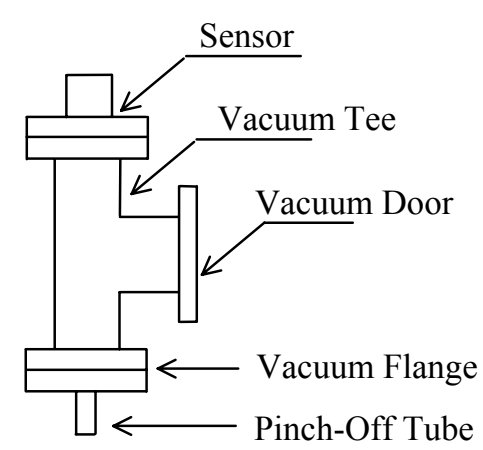

Figure 2. Schematic View of a Channel of the Ionization Gauge.

The payload has to be simplified and modeled for numerical simulations because it is impossible and unnecessary to include all the details of the payload. Figure 3 shows the modeled payload that accounts for the general flow in and around the daughter payload. The figure shows the inside by removing part of surface. The three photometer telescopes are modeled as solid cylinders and the inside region over the upper deck plate is masked. The middle deck plate is also removed for simplicity. The top channel of the ionization gauge is modeled slightly differently from the other four to account for the difference of auxiliary structures. The top channel is centered on the top plate, and the other four channels have a 45-degree deviation from the line that the photometer telescopes are 
located on. The diameter of the payload is slightly less than $0.44 \mathrm{~m}$, and its height is close to $0.75 \mathrm{~m}$. The height of the modeled payload, however, is extended to roughly $1.4 \mathrm{~m}$, because additional payload was attached to the experimental part. Nevertheless, the additional payload was not expected to affect the operation of the ionization gauges since the flight of the payload was supersonic. The ionization gauge is also simplified as shown in Fig. 4. The vacuum door is removed, and the opening has a diameter slightly larger than $0.04 \mathrm{~m}$. The vacuum tee has a diameter of $0.035 \mathrm{~m}$ and a height of $0.122 \mathrm{~m}$. The distance between the opening and the vacuum tee is about $0.058 \mathrm{~m}$.

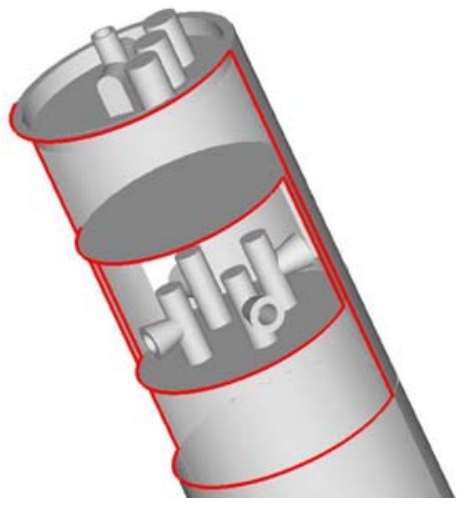

Figure 3. Modeled Daughter Payload.

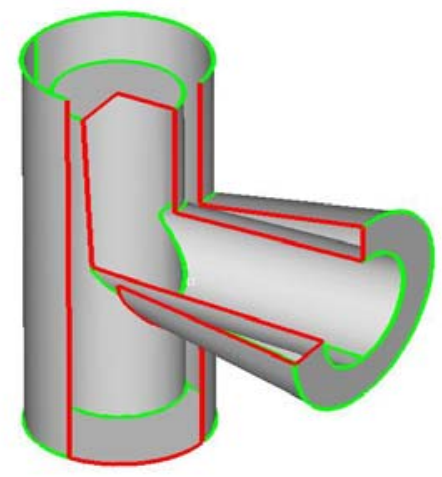

Figure 4. Modeled Ionization Gauge Channel.

\section{DSMC Simulation}

The TOMEX experiment was performed in the mesosphere and low thermosphere region. The atmosphere in this region is very rarefied. For instance, the mean free path of the atmosphere is about $0.01 \mathrm{~m}$ at $85 \mathrm{~km}, 0.1 \mathrm{~m}$ at $100 \mathrm{~km}, 1 \mathrm{~m}$ at $115 \mathrm{~km}$, and $10 \mathrm{~m}$ at $135 \mathrm{~km}$, which are all comparable to the diameter of the payload, $0.44 \mathrm{~m}$. Therefore, the direct simulation Monte Carlo (DSMC) method is used to simulate the flow.

\section{A. Direct Simulation Monte Carlo Method}

The direct simulation Monte Carlo method ${ }^{6}$ is a widely used particle method and is an effective numerical technique to simulate rarefied, nonequilibrium gas flows. It simulates rarefied gas flows based on physical principles instead of mathematical formulation. The DSMC method models gas flows through computations of motions and collisions of representative particles. The primary approximation is to uncouple the molecular motions and intermolecular collisions over small time intervals, where particle motions are modeled deterministically whereas the collisions are treated statistically.

The flow is simulated using the state-of-the-art DSMC research code MONACO. ${ }^{7}$ It is a general-purpose code for modeling 2-D, axi-symmetric, or 3D rarefied gas flows. The code is fully parallelized, and the computational domain can be automatically decomposed using the partitioning package called Metis. ${ }^{8}$ The variable hard sphere (VHS) molecular model ${ }^{6}$ is specified in this case, which assumes that the total collision cross section depends on the relative collision velocity.

\section{B. Computational Mesh}

The physics of a problem determines the required computational domain and its boundary conditions, which in this case indicates that the size of the necessary computational domain should vary with altitude for the TOMEX flows. In the DSMC simulation, the computational domain is divided into cells and filled with simulated particles where the cell size should be less than the mean free path of the atmosphere in principle. Enough cells are also required to faithfully represent the modeled payload. Besides the physical requirements, a practical restraint is to limit the total number of computational cells due to the limitation of the computer resources. For the simulated flows, small cells are used around the payload, especially for the ionization gauges, whereas relative large cells are employed for the rest of the computational domain.

Following the previous principles, several tetrahedral meshes are generated using the commercial software Hypermesh. ${ }^{9}$ A typical surface mesh is shown in Fig. 5, where the axis is assigned to the $\mathrm{Z}$ direction and the openings face the $\mathrm{X}$ or $\mathrm{Y}$ direction. The surface is modeled as a solid wall whose temperature is $300 \mathrm{~K}$ and the 
thermal accommodation coefficient is set to 1 . The entire surface is divided into 51,750 wall faces for the flow at $85 \mathrm{~km}$ and 8,134 wall faces for the flow at $120 \mathrm{~km}$. The volume of the entire computational domain is about $3.4 \mathrm{~m}^{3}$ at $85 \mathrm{~km}$, and is increased to $405 \mathrm{~m}^{3}$ at $120 \mathrm{~km}$ where the flow is subsonic. Figure 6 shows a cut-open view of the mesh for the $90 \mathrm{~km}$ case, which displays the structure of the tetrahedral mesh. The total number of tetrahedral cells varies from $1,096,235$ at $85 \mathrm{~km}$, to 141,642 at $100 \mathrm{~km}$, to 572,488 at $120 \mathrm{~km}$.

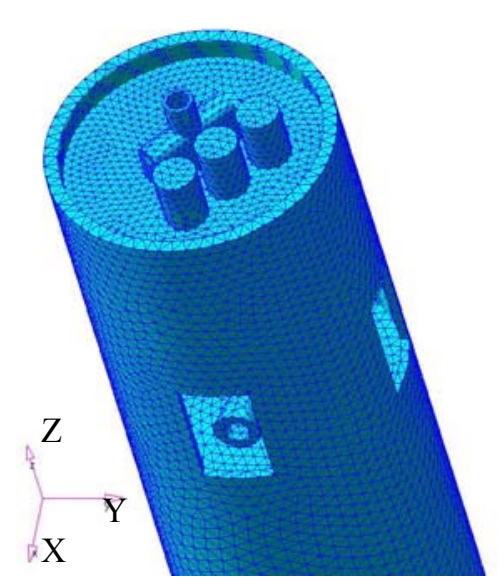

Figure 5. Surface Mesh of the Payload for the $90 \mathrm{~km}$ Case.

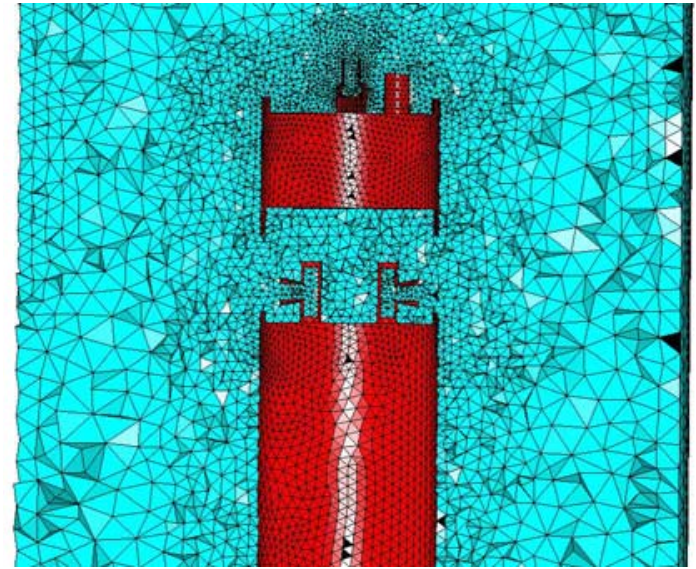

Figure 6. Cut-Open View of Tetrahedral Mesh for the $90 \mathrm{~km}$ Case.

The cell size for lower altitude simulations is not always less than the mean free path of the atmosphere. To ensure that collisions only occur between particles within a mean free path, a near-partner selection technique is used to determine the collision particle pairs. This technique is slightly different from the subcell technique where a cell is divided into several sub-cells. The near-partner technique allows a particle to collide with particles only within a small distance. In the present investigation, the distance is pre-determined and is less than half of the cell edge, which ensures that the distance is less than the local mean free path throughout the computational domain.

The payload flew at supersonic speed below $130 \mathrm{~km}$, which allows simulations to employ a small computational domain. However, a large computational domain is preferred to implement effective boundary conditions for subsonic flows experienced at higher altitude. Simulations show that the relative flow is limited to the inner part of the computational domain even when a mesh designed for the $120 \mathrm{~km}$ case is used to simulate the flow near the apogee $(143.27 \mathrm{~km})$. Hence, the meshes are acceptable for the present investigation.

\section{Particle Weighting}

In a DSMC simulation, the number of simulated particles is determined by the size of the computational domain and the number of real molecules that each simulated particle represents. For instance, the computational domain for the flow at $100 \mathrm{~km}$ has a volume of $12 \mathrm{~m}^{3}$, and the volume of the minimum cell (tetrahedral) is about $1 \times 10^{-7} \mathrm{~m}^{3}$. If 20 particles are simulated in the minimum cell, a total of $2.4 \times 10^{9}$ particles have to be simulated at the same time, where the density is assumed to be constant for the estimation. Such a simulation is extremely expensive based on the present computational ability. In order to limit the total number of simulated particles, a particle weighting strategy is employed. In general, a particle in larger cells represents more real molecules such that a similar number of particles are simulated in each cell, which is achieved by assigning a relative particle weight to each cell.

The computational cells have different particle weights for the simulated particles, which means that a particle is to be destroyed or cloned when it moves into another cell. The destroying and cloning is performed according to the probability based on the relative particle weights of the two cells. The possible effects of this particle weighting strategy on the simulations are investigated. First, several sets of particle weights are tested. One set is proportional to the cell volume, and another is proportional to the square root of the cell volume, whereas a third set allows the simulation to have roughly 20 particles simulated in each cell. Second, a similar flow having zero velocity is simulated using the particle weighting strategy. It is found that the main effect of the weight strategy is that the statistical scatter is increased due to the destroying and cloning, which means that the weighting strategy will not have serious effects on the accuracy of the simulations. 


\section{Numerical Error}

All the simulations have at least 15 particles in each cell, and the time step is less or much less than the mean collision time of the atmosphere. The total number of the simulated particles is in the order of magnitude of 10 million. A typical simulation runs for 20,000 time steps after the simulation reaches a steady state that generally takes less than 50,000 time steps, which requires approximately one day running on a 20-processor Linux cluster.

The simulation results are obtained by sampling the simulated particles, which means that the DSMC results exhibit statistical scatter. The statistical scatter can be reduced by increasing the sample size. The magnitude of the scatter, however, can only be estimated because the samples are statistically dependent and particle destroying and cloning introduce additional scatter. Based on the simulation for a zero velocity flow and a general flow running much more time steps, the statistical error for the results presented in the next section is estimated to be less than 10 percent in general, which is acceptable given the simplifications made for the geometry modeling.

\section{Results and Discussions}

Simulations are performed for the TOMEX flows at altitudes ranging from $85 \mathrm{~km}$ to $143.27 \mathrm{~km}$ (apogee). The DSMC simulations approximate the TOMEX flows as pseudo steady state flows using the flow conditions at the same altitude. The neutral temperature and densities are given by the Mass-Spectrometer-Incoherent-Scatter (MSIS) model $^{10}$ at $0957 \mathrm{UT}$ on 26 October 2000. The detailed flow conditions are listed in Table 1, where $\mathrm{H}$ is the altitude, $\mathrm{U}, \mathrm{V}$ and $\mathrm{W}$ are the velocity components of the atmosphere relative to the payload in the $\mathrm{x}, \mathrm{y}$, or $\mathrm{z}$ direction, $\mathrm{T}$ is the temperature of the atmosphere, $M$ is the Mach number of the free stream, while $\mathrm{n}_{\mathrm{O}}, \mathrm{n}_{\mathrm{N} 2}$, and $\mathrm{n}_{\mathrm{O} 2}$ are the number densities of the atmosphere component atomic oxygen, molecular nitrogen, and molecular oxygen, respectively.

Table1: Detailed flow conditions for the simulations

\begin{tabular}{ccccccccc}
\hline $\mathrm{H}(\mathrm{km})$ & $\mathrm{U}(\mathrm{m} / \mathrm{s})$ & $\mathrm{V}(\mathrm{m} / \mathrm{s})$ & $\mathrm{W}(\mathrm{m} / \mathrm{s})$ & $\mathrm{T}(\mathrm{K})$ & $\mathrm{n}_{\mathrm{O}}\left(1 / \mathrm{m}^{3}\right)$ & $\mathrm{n}_{\mathrm{N} 2}\left(1 / \mathrm{m}^{3}\right)$ & $\mathrm{n}_{\mathrm{O} 2}\left(1 / \mathrm{m}^{3}\right)$ & $\mathrm{M}$ \\
\hline 85 & 205.72 & 0 & 1050.75 & 197.4 & $5.704 \times 10^{16}$ & $1.251 \times 10^{20}$ & $3.293 \times 10^{19}$ & 3.79 \\
90 & 205.12 & 0 & 1001.60 & 188.8 & $2.463 \times 10^{17}$ & $5.546 \times 10^{19}$ & $1.432 \times 10^{19}$ & 3.70 \\
95 & 204.75 & 0 & 953.29 & 181.9 & $4.607 \times 10^{17}$ & $2.358 \times 10^{19}$ & $5.870 \times 10^{18}$ & 3.58 \\
100 & 204.37 & 0 & 902.05 & 183.2 & $4.819 \times 10^{17}$ & $9.467 \times 10^{18}$ & $2.201 \times 10^{18}$ & 3.37 \\
105 & 204.09 & 0 & 848.63 & 200.1 & $3.610 \times 10^{17}$ & $3.673 \times 10^{18}$ & $7.627 \times 10^{17}$ & 3.01 \\
110 & 203.68 & 0 & 790.99 & 236.3 & $2.347 \times 10^{17}$ & $1.470 \times 10^{18}$ & $2.614 \times 10^{17}$ & 2.56 \\
115 & 203.35 & 0 & 728.79 & 297.3 & $1.456 \times 10^{17}$ & $6.345 \times 10^{17}$ & $9.477 \times 10^{16}$ & 2.09 \\
120 & 202.98 & 0 & 660.96 & 382.8 & $9.190 \times 10^{16}$ & $3.065 \times 10^{17}$ & $3.885 \times 10^{16}$ & 1.66 \\
125 & 202.71 & 0 & 585.25 & 460.1 & $6.405 \times 10^{16}$ & $1.749 \times 10^{17}$ & $1.938 \times 10^{16}$ & 1.35 \\
130 & 202.37 & 0 & 498.51 & 523.4 & $4.787 \times 10^{16}$ & $1.112 \times 10^{17}$ & $1.114 \times 10^{16}$ & 1.09 \\
135 & 201.97 & 0 & 393.48 & 579.1 & $3.722 \times 10^{16}$ & $7.539 \times 10^{16}$ & $7.009 \times 10^{15}$ & 0.84 \\
140 & 201.69 & 0 & 247.11 & 628.0 & $2.980 \times 10^{16}$ & $5.348 \times 10^{16}$ & $4.701 \times 10^{15}$ & 0.58 \\
143.27 & 201.50 & 0 & 1.87 & 656.7 & $2.612 \times 10^{16}$ & $4.357 \times 10^{16}$ & $3.717 \times 10^{15}$ & 0.36 \\
\hline
\end{tabular}

\section{A. Flow Patterns}

The TOMEX flight was expected to generate complicated flow structures during the experiment. Figures 7-12 illustrate the flow patterns predicted by the DSMC simulation when the altitude is $85 \mathrm{~km}$, where the Mach number of the free stream relative to the payload is roughly 3.8. A strong bow shock wave is observed (Figs. 7-9) about $10 \mathrm{~cm}$ away from the payload. The bow shock wave is nearly symmetric on the $\mathrm{x}=0$ plane although the payload is unevenly distributed in the front, whereas it is non-symmetric in the $y=0$ plane because of the 11-degree angle of attack. The pressure behind the shock wave is increased due to the shock compression. The increase reaches the maximum on the top of the payload and decreases downward along the payload surface. The 11-degree angle of attack of the flow also means that the side of the payload facing toward the flow has a larger pressure and the opposite side has a lower pressure. Relatively low pressure is predicted inside the payload. The pressure in the side channels of the ionization gauge depends on whether the opening faces the flow. The channel in the negative $\mathrm{x}$ direction has a large pressure and the channel in the positive $\mathrm{x}$ direction has a smaller pressure, whereas the two channels located in the $\mathrm{x}=0$ plane agree within numerical error. The pressure contours plotted in Fig. 10 show the overall pressure distribution on the payload surface. Figures 11 and 12 show the density and temperature contours in the $y=0$ plane. The density plot is more similar to the pressure plot as compared with the temperature plot because the temperature on the surface is kept at $300 \mathrm{~K}$. It is also found that there is a large temperature jump around the outer surface of the payload although the Knudsen number of the flow evaluated as the ratio of the mean free path of the free stream to the diameter of the payload is only 0.02 . 


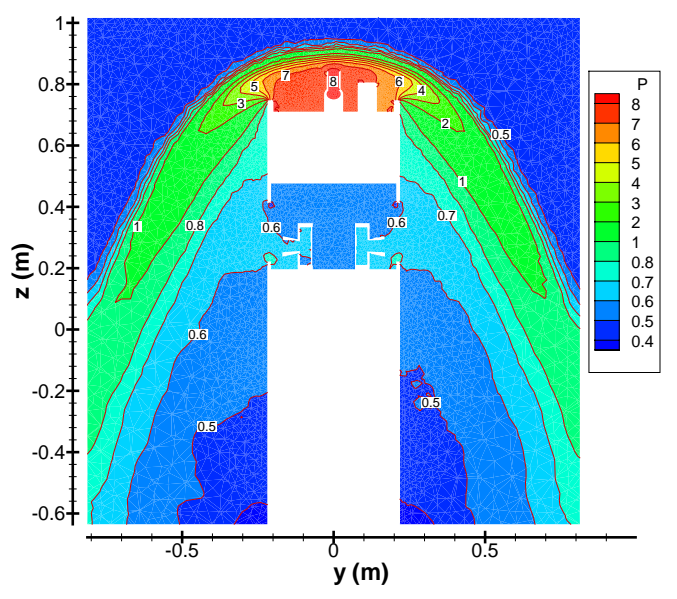

Figure 7. Pressure (Pa) Contours in $x=0$ Plane at $85 \mathrm{~km}$ Predicted by DSMC Simulation.

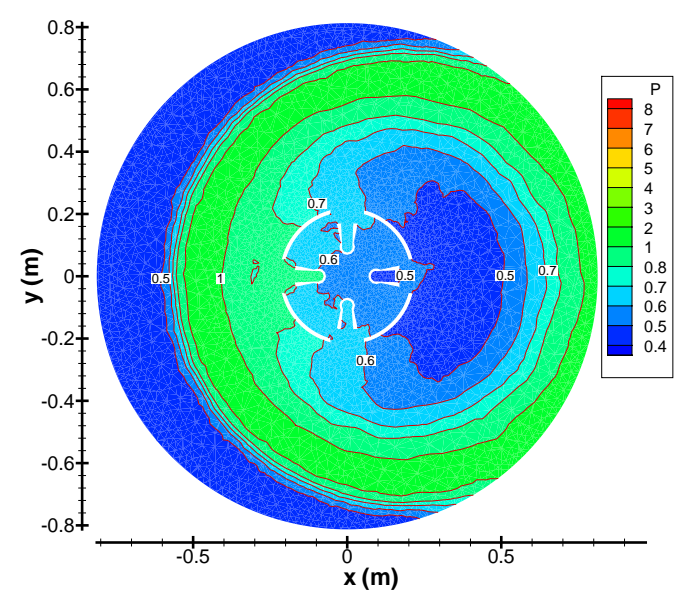

Figure 9. Pressure (Pa) Contours in $z=0.275$ Plane at $85 \mathrm{~km}$ Predicted by DSMC Simulation.

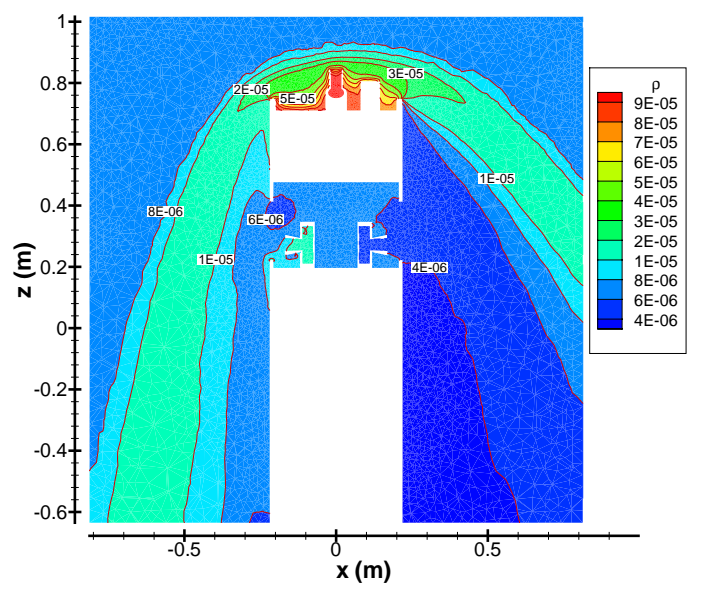

Figure 11. Density $\left(\mathrm{kg} / \mathrm{m}^{3}\right)$ Contours in $y=0$ Plane at $85 \mathrm{~km}$ Predicted by DSMC Simulation.

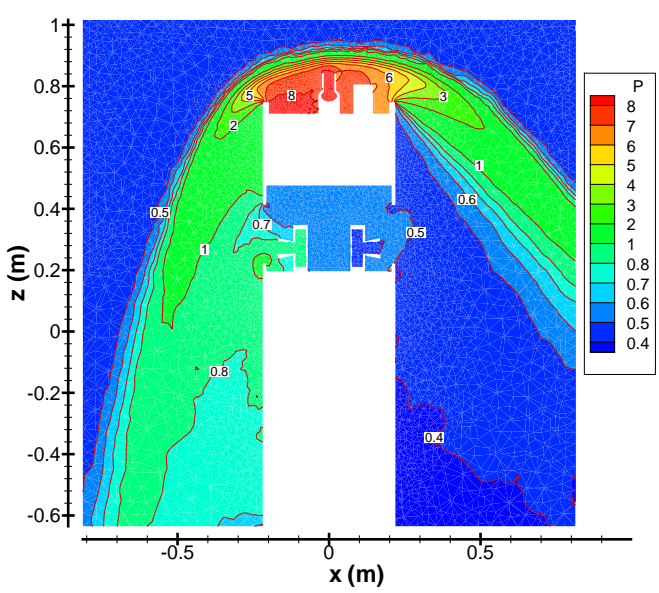

Figure 8. Pressure (Pa) Contours in $y=0$ Plane at $85 \mathrm{~km}$ Predicted by DSMC Simulation.

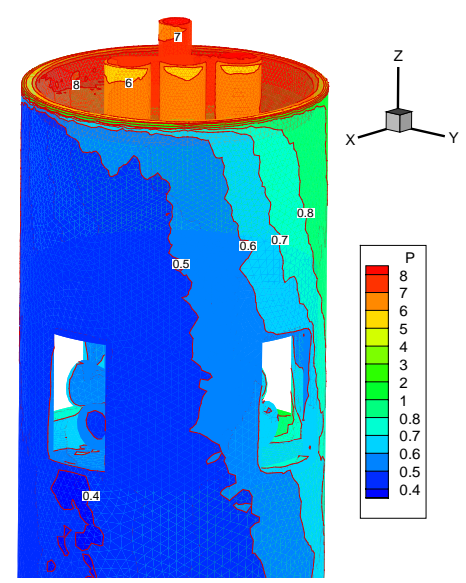

Figure 10. Surface Pressure (Pa) Contours at $85 \mathrm{~km}$ Predicted by DSMC Simulation.

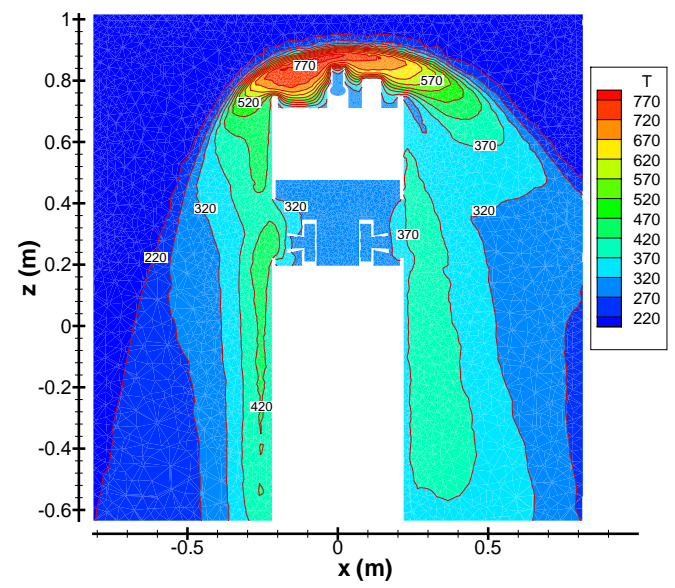

Figure 12. Temperature (K) Contours in $y=0$ Plane at $85 \mathrm{~km}$ Predicted by DSMC Simulation. 


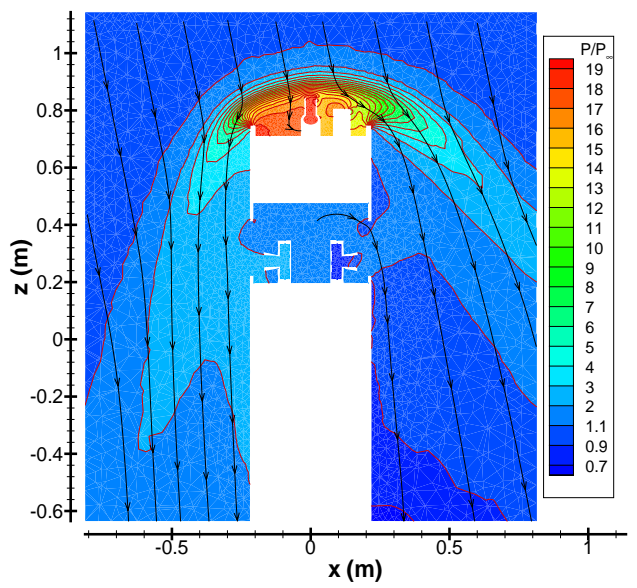

Figure 13. Pressure Contours in $y=0$ Plane at $90 \mathrm{~km}$ Predicted by DSMC Simulation.

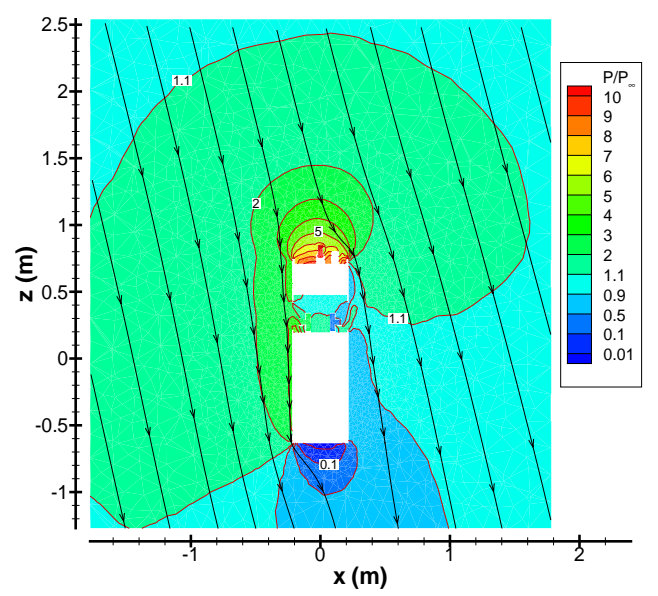

Figure 15. Pressure Contours in $y=0$ Plane at $110 \mathrm{~km}$ Predicted by DSMC Simulation.

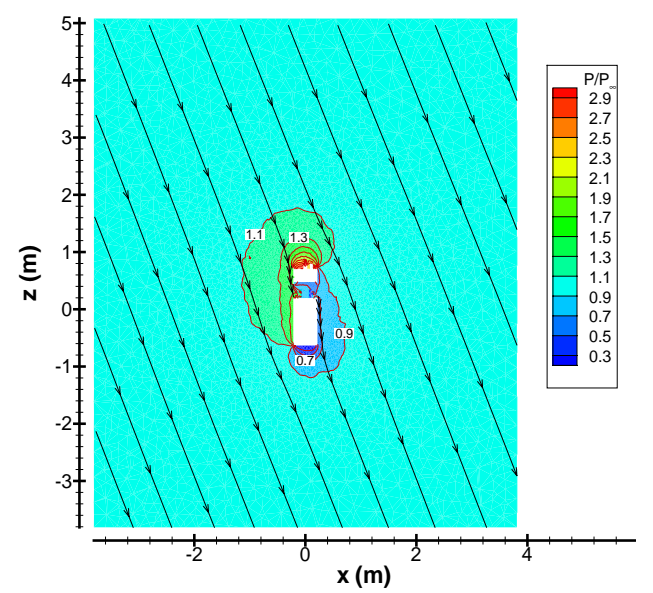

Figure 17. Pressure Contours in $y=0$ Plane at $130 \mathrm{~km}$ Predicted by DSMC Simulation.

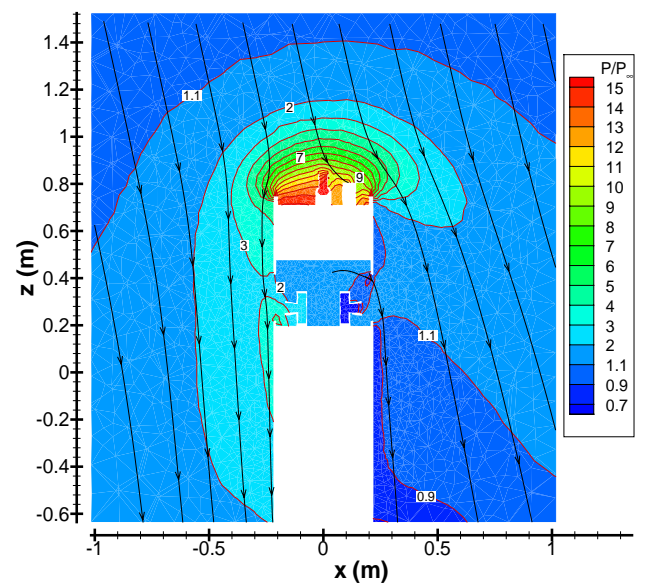

Figure 14. Pressure Contours in $y=0$ Plane at $100 \mathrm{~km}$ Predicted by DSMC Simulation.

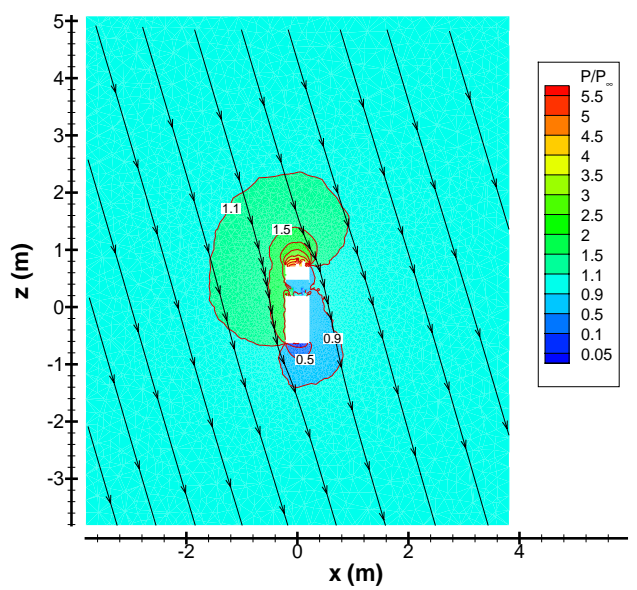

Figure 16. Pressure Contours in $y=0$ Plane at $120 \mathrm{~km}$ Predicted by DSMC Simulation.

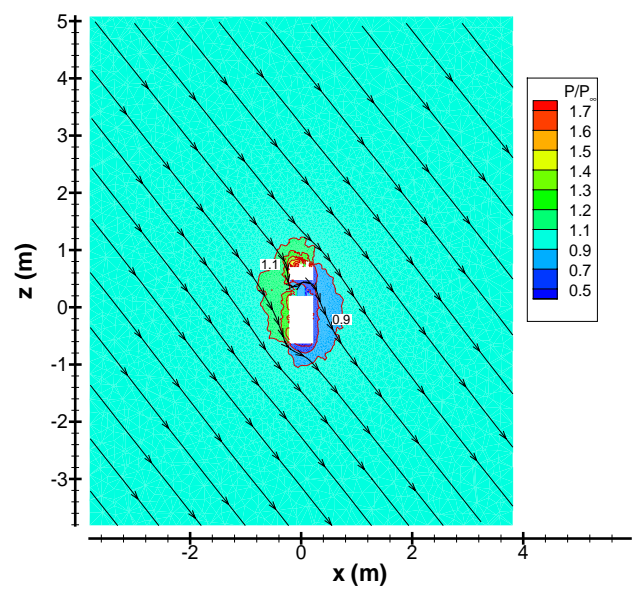

Figure 18. Pressure Contours in $y=0$ Plane at $140 \mathrm{~km}$ Predicted by DSMC Simulation. 
The flow patterns vary with altitude due to changing flow conditions. First, the mean free path of the atmosphere is increased by three orders of magnitude from $85 \mathrm{~km}$ to $143 \mathrm{~km}$, which changes the flow from the near-continuum regime to the free molecular regime. The thickness of the bow shock wave if it exists will also increase because the thickness of a normal shock wave is roughly several mean free paths, which means that a shock wave in a rarefied condition can extend over a large distance. Also, the Mach number of the free stream is decreased from supersonic to subsonic. For subsonic flows, the effects at any location are global.

Figures 13 to 18 illustrate the flows at several altitudes. Each figure shows the pressure contours normalized by the free stream pressure and the stream lines in the $y=0$ plane where the velocity in the $y$ direction is very small. As shown, a shock-like structure is still observed for the flow at $90 \mathrm{~km}$, whereas a shock wave is hardly recognized for the flow at $100 \mathrm{~km}$. The pressure plot for the flow at $110 \mathrm{~km}$ resembles that for the subsonic flow at $140 \mathrm{~km}$ although the free stream Mach number is roughly 2.5. It is also found that the pressure variation decreases as the altitude increases, and the variation is limited to a relatively small region at higher altitude.

\section{B. Pressure in the Ionization Gauges}

The ionization gauge measures the neutral density of the gas in the vacuum tee, whereas the pressure is usually used to compare with data obtained using other techniques. Therefore, the pressure in the vacuum tee is analyzed before the simulation results are compared with the measurement data.

The inviscid compressible flow theory can predict the total pressure using the Mach number of the free stream: ${ }^{11}$

$$
\frac{p_{0}}{p_{\infty}}=\left(1+\frac{\gamma-1}{2} M_{\infty}^{2}\right)^{\frac{\gamma}{\gamma-1}}
$$

where $p_{0}$ is the total or stagnant pressure, $p_{\infty}$ is the free stream pressure, $M_{\infty}$ is the free stream Mach number, and $\gamma$ is the ratio of specific heats. For subsonic flows, $p_{0}$ can be regarded as the pressure in the vacuum tee of the top channel because there is no flow in the vacuum tee. For supersonic flows, however, the total pressure will drop across the shock wave. The total pressure behind the shock wave or the pressure in the vacuum tee, $p_{02}$, is approximated following the normal shock wave relations as follows: ${ }^{11}$

$$
\frac{p_{02}}{p_{0}}=\left[\frac{2 \gamma M_{\infty}^{2}-(\gamma-1)}{\gamma+1}\right]^{-\frac{1}{\gamma-1}}\left[\frac{(\gamma+1) M_{\infty}^{2}}{2+(\gamma-1) M_{\infty}^{2}}\right]^{\frac{\gamma}{\gamma-1}}
$$

The pressures in the side channels are generally less than the total pressure because of the viscous interactions between the flow and the surface of the payload.

The pressure in the vacuum tee can also be approximately predicted by the free molecular flow theory because TOMEX flows at high altitude are already in the free molecular flow region. The expression based on the free molecular theory is as follows: ${ }^{12}$

$$
\begin{gathered}
p_{f}=p_{\infty}\left\{g\left(s_{3}\right) e^{-s_{3}^{2}}+\left[1 / 2+\sqrt{\pi} s_{3} g\left(s_{3}\right)\right]\left[1+\operatorname{erf}\left(s_{3}\right)\right]\right\} \\
g\left(s_{3}\right)=\frac{s_{3}}{\sqrt{\pi}}+\frac{1}{2} \sqrt{\frac{T_{r}}{T_{\infty}}}, \quad s_{3}=u_{3} \sqrt{\frac{m}{2 k T_{\infty}}}
\end{gathered}
$$

where $p_{f}$ is the pressure in the vacuum tee, $T_{\infty}$ is the free stream temperature, $T_{r}$ is the reflected temperature (it is assumed as the wall temperature), $m$ is the average molecular mass, $k$ is the Boltzmann constant, $u_{3}$ is the velocity component normal to the door of the channel, and $\operatorname{erf}()$ is the error function. 
The pressure in the top channel of the ionization gauge is plotted in Fig. 19. It shows that the pressure predicted by the DSMC simulation is very close to that obtained using the compressible flow theory when the altitude is low, and it is a little smaller than that predicted by the free molecular theory when the altitude is high. Figure 20 shows the pressure in the side channels of the ionization gauge. The DSMC pressure in the channel facing the flow, $\mathrm{P}_{-\mathrm{x}}$, is about $20 \%$ lower than that predicted by the free molecular theory, which may be due to the effects of the existence of the payload surface. For the pressure in the channel opposing the flow, $\mathrm{P}_{+\mathrm{x}}$, the agreement between the DSMC results and those predicted using the free molecular theory is good when the altitude is high, and the large difference at low altitude indicates that the free molecular theory is invalid here although the pressures at the other channels seem close to the DSMC results.

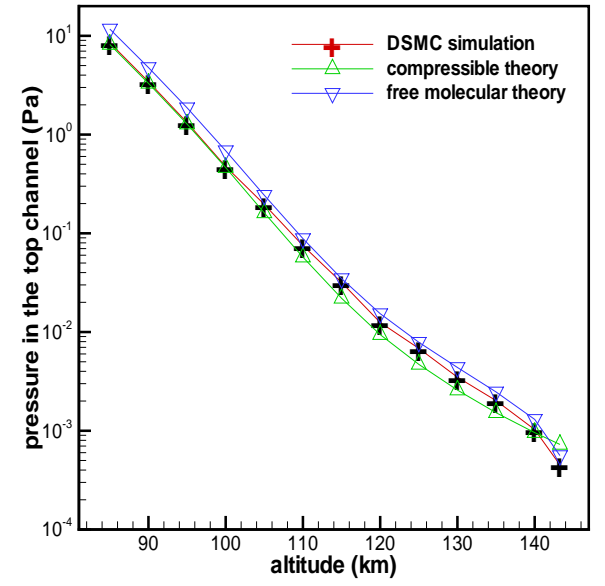

Figure 19. Pressure in the Top Channel

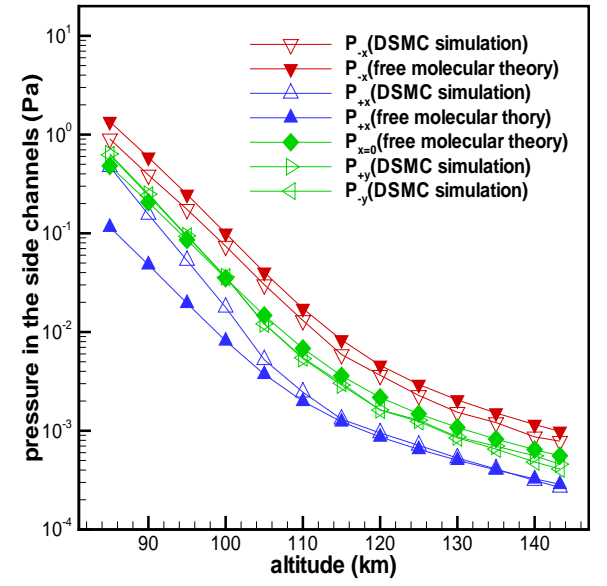

Figure 20. Pressure in the Side Channels

\section{Pressure Comparison between Simulation Results and Measurement Data}

The computed pressures in the channels of the ionization gauge are compared with the measurement data. During the TOMEX experiment, the payload had a spin rate of almost exactly $1 \mathrm{~Hz}$, which means that each of the side channels recorded the pressure that had a pseudo-frequency of $1 \mathrm{~Hz}$.

Figure 21 shows the pressure in the channels of the ionization gauge obtained from the DSMC simulations and the measurement. All the measurement data has been calibrated and adjusted to account for effects such as gas venting in the ionization gauge. The simulation offers good agreement with the top channel calibrated data expect at the lowest altitude. The calibrated side data shown in the figure is the measurement pressure in one of the side channels of the ionization gauge. The measurement pressures in all side channels are very similar, and their average is also plotted in the figure. It is found that the DSMC simulation predicts a maximum pressure in the channel facing the flow and a minimum pressure in the channel opposing the flow, and these pressures bound the measurement data.

The slight difference between the simulation results and the measurement data is not surprising accounting for possible differences of the flow conditions and payload configuration. First, the physical configuration of the payload is much more complicated than is modeled. There are many un-modeled auxiliary parts and all kinds of cables. For instance, the middle

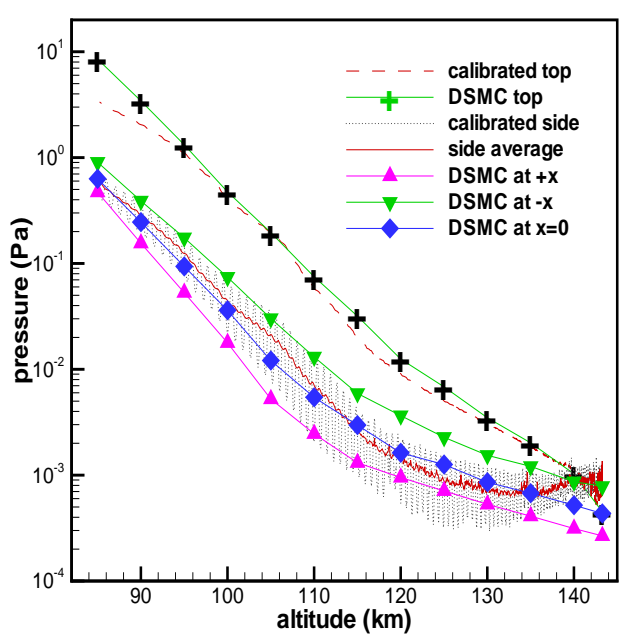

Figure 21. Pressure Comparison between DSMC Simulation Results and Calibrated Measurement Data.

deck plate and the vacuum doors of the channels of the ionization gauge are removed in the simulation. Second, the flight is actually unsteady, which may introduce differences when the flow is treated as a pseudo-steady state at each altitude. Third, the atmosphere conditions used in the simulation are based on the MSIS model. The difference between the model data and the real situation may account for some difference. The data obtained from the lidar 
measurement do indicate that the temperature of the atmosphere below $92 \mathrm{~km}$ was a little warmer than that given by the MSIS model. For instance, the pressure in the top channel will decrease roughly $16 \%$ if the pressure of the atmosphere is kept the same while the temperature predicted by the lidar measurement is used in the simulation. The decrease of the channel pressure is because the free stream Mach number is decreased due to the warmer atmosphere. Accounting for all these factors, the DSMC simulations are very successful in studying the TOMEX flows, and show flow details that are not revealed by the measurement.

\section{Conclusion}

The rarefied, three-dimensional flows experienced during the Turbulent Oxygen Mixing Experiment (TOMEX) at altitudes between $85 \mathrm{~km}$ and $143 \mathrm{~km}$ were simulated using the direct simulation Monte Carlo (DSMC) method. The details of important geometric structures of the payload were modeled, and several complex tetrahedral meshes were generated for the simulations.

The DSMC simulations employed a particle weighting technique to limit the total number of simulated particles and a near-partner selection strategy to determine the collision particle pairs. Simulations showed that a bow shock wave was generated in front of the payload at low altitude that became diffusive when the altitude was increased even when the Mach number of the free stream was larger than 2.5. It was also found that the pressure in the channels of the ionization gauge decreased monotonically with increasing altitude where the pressure variation was also decreased.

The pressure at several locations was estimated using compressible flow theory and free molecular flow theory, and indicated that the DSMC simulations were reasonable. Comparison between the DSMC results and the measurement data showed that the DSMC simulations predicted the TOMEX flows very well. The accuracy of the computations indicates that DSMC should be used to design future flight experiments.

\section{Acknowledgments}

This work is funded in part by NASA through grants NAG5-5235 (TOMEX) and NAG5-5412 (TRIO). The authors are also grateful for computational resources provided on the Maui High Performance Computing Center and on the high performance clusters in the Department of Aerospace Engineering and Mechanics at the University of Minnesota.

\section{References}

${ }^{1}$ Hecht, J. H., Liu, A. Z., Bishop, R. L., Clemmons, J. H., Gardner, C. S., Larsen, M. F., Roble, R. G., Swenson, G. R., and Walterscheid, R. L., "An Overview of Observations of Unstable Layers During the Turbulent Oxygen Mixing Experiment (TOMEX)," Journal of Geophysical Research, Vol. 109, D02S01, 2004.

${ }^{2}$ Liu, A. Z., Roble, R. G., Hecht, J. H., Larsen, M. F., and Gardner, C. S., "Unstable Layers in the Mesopause Region Observed with $\mathrm{Na}$ Lidar during the Turbulent Oxygen Mixing Experiment (TOMEX) Campaign," Journal of Geophysical Research, Vol. 109, D02S02, 2004.

${ }^{3}$ Bishop, R. L., Larsen, M. F., Hecht, J. H., Liu, A. Z., and Gardner, C. S., “TOMEX: Mesospheric and Lower Thermospheric Diffusivities and Instability Layers," Journal of Geophysical Research, Vol. 109, D02S03, 2004.

${ }^{4}$ Hecht, J. H., Liu, A. Z., Walterscheid, R. L., Roble, R. G., Larsen, M. F., and Clemmons, J. H., "Airglow Emissions and Oxygen Mixing Ratios from the Photometer Experiment on the Turbulent Oxygen Mixing Experiment (TOMEX)," Journal of Geophysical Research, Vol. 109, D02S05, 2004.

${ }^{5}$ Larsen, M. F., Liu, A. Z., Bishop, R. L., and Hecht, J. H., “TOMEX: A Comparison of Lidar and Sounding Rocket Chemical Tracer Wind Measurements," Geophysical Research Letters, Vol. 30, No. 7, 2003, pp. 1375-1378.

${ }^{6}$ Bird, G. A., Molecular Gas Dynamics and the Direct Simulation of Gas Flows, Oxford University Press, 1994.

${ }^{7}$ Dietrich, S., and Boyd, I. D., "Scalar and Parallel Optimized Implementation of the Direct Simulation Monte Carlo Method," Journal of Computational Physics, Vol. 126, 1996, pp. 328-342.

${ }^{8}$ Karypis G., and Kumar, V., "METIS, A Software Package for Partitioning Unstructured Graphs, Partitioning Meshes, and Computing Fill-Reducing Orderings of Sparse Matrices, version 4.0," Technical Report, University of Minnesota, Department of Computer Science and Engineering, 1998.

${ }^{9}$ Hypermesh, Version 5.0, Altair Engineering, Inc., Troy, Michigan.

${ }^{10}$ Hedin, A. E., "Extension of the MSIS Thermospheric Model into the Middle and Lower Atmosphere," Journal of Geophysical Research, Vol. 96, 1159, 1991.

${ }^{11}$ Anderson, J. D., Modern Compressible Flow: with Historical Perspective, McGraw-Hill Press, 1990.

${ }^{12}$ Gombosi, T. I., Gaskinetic Theory, Cambridge University Press, 1994. 